\title{
ApPlication Of AugMENTED REAlity \& VIRTUAL REALITY IN ARCHITECTURE AND PLANNING: AN OVERVIEW
}

\author{
Ms. Pearl Jishtu ${ }^{1}$ and Madhura A Yadav \\ ${ }^{1}$ School of Architecture \& Design, Manipal University Jaipur \\ Dehmi Kalan, Off Jaipur-Ajmer Expressway, Jaipur - 303 007, \\ Rajasthan, India \\ ${ }^{2}$ Manipal University Jaipur \\ Dehmi Kalan, Off Jaipur-Ajmer Expressway, Jaipur - 303 007, \\ Rajasthan, India
}

\begin{abstract}
$A R$ and $V R$ - simulation tools created to assist global evolution for saving time. Time as resource is difficult to harness; however, it would make work highly efficient and productive when tackled with automation. All concerned are excited about AR and VR's involvement in our lifestyle, but not all have comprehended its impact. AR and VR in Architecture \& Planning were introduced as assisting tools and has helped generate multiple design options, expanded possibilities of visualization, and provided us with more enhanced, detailed, and specific experience in real-time; enabling us to visualize the result of work at hand well before the commencement of the project. These tools are further being developed for city development decisions, helping citizens interact with local authorities, access public services, and plan their commute. After reviewing multiple research papers on AI, it was observed that all are moving forward with the changes brought by it, without entirely understanding its role. This paper provides an overview of the application of $A R \&$ $V R$ in architecture and planning.
\end{abstract}

\section{KEYWORDS}

Virtual reality (VR), Augmented reality (AR), Architecture, Urban Design, Human Computer Interface (HCI)

\section{INTRODUCTION}

Architecture and urban design rely on a similar process that includes data collection and analysis, design decision, visualization, and evaluation. AR has been developed for immersive and collaborative use in all of these stages to analyze the best design solution outcome in a better manner. Some of these processes are already in the application. Apart from using the simulation for the professionals, the application of these systems is also being evolved for the use of the public, students in the field of design, and policy makers. A study of various research papers was conducted and summarized to review and analyze the current developments in simulated or algorithmic tools. The papers cover diverse topics under theory, concept, systems, tools, applications, and implications. 


\section{Methodology}

The research paper is a summarization report of utilization of AR (Augmented Reality) and VR (Virtual Reality) in the field of architecture and urban design, for an in-depth understanding of the potentials and challenges of the technology. The main objective of the research is to record the progress of these tools with a perspective specific to the design field and to understand its diverse applications. The information was compiled by going through 20 research papers on the subject cited, of various scholars, published in conferences and readily available on the research websites. The keywords were used to search for the subject related papers and were then studied in depth for a better understanding of the same.

\section{LiteratURE STUDY}

(Seichter et al. 2005) ${ }^{[5]}$ Elaborated on understanding the association between human beings, the natural world and the successive insinuation on their interaction. This was related to Phenomenology, which is also related to the realm of Human-Computer Interfaces (HCI) and urban design. Phenomenology is the principle of empirical observation on events that are based on different theories but cannot be directly explained through them. Various theories have been put forth about the connection between perceivably real and virtual objects. Husserl and Gibson (1931) ${ }^{[1]}$, Schutz and Northwestern University (1967) ${ }^{[2]}$ and Norman (1988) ${ }^{[3]}$ came up with theories elaborating the intangible connections between real and virtual objects, to establish a conscious sense of presence.(i) Human Computer Interfaces (HCI), of which AR and VR are the components, having a great scope in the field of urban design and architecture (Frazer et al. $1980)^{[4]}$. This allows the user to work in both real and virtual environments simultaneously within all dimensions, instead of the traditional physical models. Their inherent physical nature makes them easy to understand by both the layman and experts alike. However, they find limitations in their flexibility towards a dynamic presentation. Augmented Reality (AR) however, offers a variety of opportunities where urban design can gain from in the intersection of real and virtual realms. (ii) Collaboration is important in urban designing, and cognition being another feature is invoked through the utilization of immersive technology and perceptible interlinking. Sensations triggered by 'grasping' and 'moving' aid the designers' cognitive process and their awareness of 'being inside', which in AR, is a mixture of real and virtual(iii) Cognition is the ability to comprehend and create comparison between data through analysis. Thus the understanding of the design and its relationship within the urban context is enhanced, site-specific contexts are better recognized, and various options can quickly be investigated. Since AR permits a blending of virtual entities with a real environment, it can, as a result, increase the comprehension of the design proposal. (iv) Innovation- Students and teachers can explore various theoretical and practical frameworks to understand dynamically and spatially complex relationships.

One of the study goals was to identify how designers create and communicate initial design ideas by employing AR. Using an AR system, designers gain a more complex understanding of their design relationships and engage in a richer communication with their partners. AR contributes to urban design in an innovative approach, thus enabling new forms of design expressions.

(Sørensen, 2006) ${ }^{[6]}$ States that Augmented Reality is a mediating tool to design ideas between the design and a person. It elaborates upon the components of the AR system in principles, which include; (i) Position registration system to provide the exact geo-location for the placement of the 3D model. For interiors, infrared, ultrasound systems, or graphical markers system are explored with limited installation time. While the use of the exterior of differential GPS is accurate, the system is currently expensive, (ii) Orientation registration system - This requires the user's exact location and the user's angle. Currently this has limitations of a slow data processing, resulting in 
lagging and inaccuracies in reading data leading to disproportionate models in the surrounding, and (iii) Display device - there are multiple viewing options, one of them being a headset, which currently has two different systems of operating. Ones consist of a transparent screen with the model visual on it and in the other the surrounding images are fed to the AR system without a transparent screen.

The limitations analysed of AR Systems in Architecture and Urban Design were, minor reception of the system's markers leading to poor visualisation due to the sun glare and in case of dynamic AR systems, the accurate positioning by the system leads to errors like disproportionate models. AR will induce new insights in architectural learning, introducing the students to new parameters that will enable them to immerse in their designs virtually. This will enable them to study alternatives at different abstraction levels, thus contributing to the earlier design phases better. Dynamic models and collaborative systems will broaden the scope, and it will be possible to change proposed structures or models in AR, and one can experience the simulation of changes in physical conditions, animate, and visualize pollution, noise, etc.

(Döllner et al. 2006) ${ }^{[7]}$ Virtual city 3D models are integerated comprehensive systems utilizing diverse urban space information. The application of this standardised model is discussed in the perspective of various feilds that require visual representation of urban environment. The model is a virtual representation of terrain, building and vegetation models alongwith roads/transportation systems. It aims to incorporate three categories of data (i) Cadastral data delivers the footprints of buildings and land ownership along with the ownership status, (ii) Digital terrain model and photovisualisation, (iii) 3D Building model geometry captured by laserscanning and photogrammetry based methods, and (iv) architectural models with their elements and historic references. There is no widely accepted standardized system but some of the systems in use include: CityGML, 3D Studio Max object files and VRML files, ESRI Shapefiles. The system is an important collaborative tool and is a great support system for 3D decision making. It covers functionality like managing, integrating, and distributing complex geoinformation supported a consistent communication metaphor, the virtual 3D city model. These functions are then divided up in sub-systems for content authoring, editing, storing, and presentation extendible 3D city model system. In addition, techniques for the automated mapping of 2D landscape plans and architectural plans to 3D geovirtual environments based on a heuristic-algorithm approach are investigated.

(Seichter, 2007) ${ }^{[8]}$ Explored the aspects of co-located collaborative design process with the utiliztion of 3D models and AR tools. Experiments conducted to record the user response of these tools in the field of design communication, physical engagement and the concept of presense. Factors of tangibility and directness of the user input device were used to distinguish the two experimental conditions. The observations suggested that the involvement of new techniques and tools for a better understanding of the inter-spatial relation are beneficial for design, with a better and an effective output. The adoption of novel methods like the TUI keeps on linking the bridges between reality and virtuality. The investigation has raised scopes for the future development to incorporate these technical interfaces to achieve a collaborative settting with a consideration for presence and human perspective.

(Wang, 2009) ${ }^{[9]}$ Discusses the relation between MR (Mixed Reality), VR (Virtual Reality) and AR (Augmented Reality), while futher elaborating upon their potenials, applications and challenges in the field of architecture and design. Some of the software and hardwares were disscused with their current application for the various stages of design were Sketchhand+, Benchworks, ARTHUR, AR CAD (Augmented Reality Computer Aided Design), MRCVE (Mixed Reality-based Collaborative Virtual Environment), Studierstube, BUILD IT and TINMITH2. Even though there is plenty of research and development in the field of AR and VR 
simulations, but for specific application like architecture and urban design the field is yet to be explored in detail. The main reasons of this stagnation are (i) lack of industrial domain knowledge, due to the inavailibility of a well organised integerated 3D database providing information of the mechanical services, (ii) creating a well defined reality model, and (iii) the accuracy and the robustness of the current tracking systems.In brief AR system is not very flexible and specific in current use and needs a pre analysis of the environment. The future robust $\mathrm{AR}$ aims to have a system that could work in unstructured indoor and outdoor environments.

(Abboud, 2014) ${ }^{[10]} \mathrm{AR}$ and VR has a wide range of toolsets that help generate simulations, out of these subsets the mobile augmented reality (MAR) is one option which is widely explored in practical research. The MAR system has diverse applications across design, construction and post construction that provides us with in-depth data. These include (i) interaction of the clients, consultants and designers at the initial design stage, (ii) the BIM data can be directly overlaid at the site, to oversee any later stage issues and it could be efficient in locating materials, equipment and safety zones. It could assist the labours to carry out complex work by aiding them with insitu visual assistance. On site model work progress could also be looked over and reported to the site, preventing causalities, and (iii) Post-Completion the facilities to maintain the building structure can take aid of the augmented system to locate the services and facilitate the maintenance requirements. These MAR applications face technological, social and financial issues which need to be resolved before moving forward. Tracking systems need to be evolved and specific for locating services, specialists will have to train the workers with these systems and new protocols will have to be implemented for maximum efficiency.

(Seichter, 2020) ${ }^{[11]}$ Explores the visualisation of urban data beyond the current 2D use, incorporating the tangible and non-tangible features which are essential to understand the human interaction in an urban space. The interaction techniques and technologies incorporate the significant contribution of BenchWorks that is collaborative and immersive AR-enabled working environment. BenchWorks is developing the tools and technologies to provide an immersive AR experience specifically for Urban Design data collection and analysis. Their Benchmark sketchbook+ has turned out to be a very useful tool for the initial design decision making process. ARToolKit ${ }^{[12]}$ provides an easy way to create input devices that do not rely on wires and therefore are more convenient in a multi-user setup. The magnet tracking provides higher precision for head orientation and, consequently, provides a more stable augmented vision. Major benefits for this early prototype are combined: the precision of a magnetic tracking system and the freedom of tangible interfaces overcome occlusion within the scene.

Shared Tangible City - Choice and combination of media play a key role in the investigation of space and, subsequently, architecture. BenchWorks goes beyond that, creating a toolkit for investigating a site's spatial feature dynamically in a setting with local and remote collaboration capabilities. The way a user can design in BenchWorks is by representation of void and non-void space of the city, adding volumes and making notes; the interaction being tight-knit with tangible interfaces. The toolkit uses two different tools: toolbox and the modifiers represented by pens and the sponge.

(Kim et al. 2011) ${ }^{[13]}$ used an AR system in a studio setting to introduce shared learning as an essential part in urban design, where students are conversant in syntax and grammar. AR is employed to dynamically and spatially understand complex relationships, which helps people understand urban issues during a new and creative way. The experiment conducted helped to understand the participants' sense of presence and their level of immersion. AR conceptualisation gave an in-depth understanding of the algorithmic framework and modelling that are adopted and the various challenges that are to be faced just like the sun glare (Chung et al. 2009) ${ }^{[14]}$, temporary lags created in the systems (Wang and Dunston 2005) ${ }^{[15]}$, heavy equipment (Santos et 
al. 2007; Chung et al. 2009) ${ }^{[16][14]}$ and therefore the human factor. These concepts and ideas implemented with hardware or software tools that have their own challenges like(i) the human interaction is not very experiential, (ii) potential use of agent-based is yet to be explored (Belcher and Johnson 2008) ${ }^{[17]}$, and (iii) portability of the entire system is still a substantial limitation. While evaluating the whole system for the quality and the productivity through quantitative survey, cognitive and effective workability were the common issues noted. Even though a lot of literature survey and practical study was done on this subject, a wide range industrial adoption was still found lacking. This was mainly due to the expensive and heavy toolkits that were not designed specifically for the design field.

(Schubert et al. 2015) ${ }^{[18]}$ Design Communication in the form of $2 \mathrm{D}$ drawings and details is only comprehendible by the professionals in the field, but not the clients or the laymen constructing the structure. Design decision making at the initial stages becomes easier with illustration drawings which are currently used in the digital format. Now, with the intervention of AR and VR systems it would bring better results. The idea would be to simulate the master plan or the building geometry on a table top VR or could also be augmented on the site via headset. The two design components discussed are the collaborative design platform and the on-site ARapplications. The second is discussed on the basis of its potential to grow and evolve, as it is bound to make certain processes of design better.

(Thabet, 2002, updated 2015) ${ }^{[19]}$ The foremost important benefit of VR is that, the clients can rehearse a building's design experiencing the finished product as if they were in the actual space. Flaws within the designs can also be detected easily with VR. Certain concerns raised were, (i) use of 3D and VR are often beneficial as long as used early at the conceptual design process, (ii) housing developers did not want to expose users to views from sensitive unrealistic angles, and (iii) when dealing with local authorities to avoid exposing the design or construction to other issues where an advanced visualization must be used with care. The workers can utilize AR to detect hazardous faults and prevent causalities. Retik and VIRCON are two automated simulations that deal within the constructional perspective. The complete analysis of the structure just in case of calamities could be conducted with the assessment of the relief shelter within the particular building. The utilisation of these tools is to simplify the upcoming complex designs.

(Milovanovic, 2017) ${ }^{[20]}$ Highlighted two aspects of AR and VR design i.e., interaction and immersion. The article in the initial portion discusses the analysis derived from research papers regarding the development of $\mathrm{AR}$ and $\mathrm{VR}$ in the various design processes and its subsequent implementation. It was observed that maximum research papers were about the development of the hardware/software development of the system, and then followed by design; however, the applications are very few to date. It helps us analyze and understand that due to certain limitations like the cost of technology, we are stuck at the conceptual and design stage. The five significant systems elaborated upon this text include; (i) HDM AR- as proposed by the Benchmark system, (ii) HYVE 3D - a system that is an interplay of Computer-Aided Design (CAD) and Virtual Reality, resulting in the formation of VRAD (Virtual Reality Aided Design), making collaborated design in the initial stages feasible, (iii) HMD VR, used in the CAP VR system - The environment proved to enhance students' design quality. Students' immersion in the virtual model of their design augmented their spatial comprehension and enriched their evaluation. However, the communication with the tutors was not seamless because students were wearing an HMD, (iv) Tangible AR - the system illustrated by the Luminous Planning Table, and (v) SDAR or SAR - It utilizes the joint surfaces available to us around like the walls and floors to be used as display devices, providing the design on a 1:1 scale experience.

(Broschart, 2015) ${ }^{[21]}$ Studies the varied tools that are being used or being developed for the use of urban planning and architectural visualization. Keeping in mind the various limitations that are 
foreseen with the use of AR systems (portability of computers and time required for installation), this research lays focus on the smart portable i.e., the mobile phone which consists of fourelement components (1) Computer software generating the 3D model that is to visualized, (2) Geo locating and extracting the exact location data, (3) detecting the user's orientation and (4) the last would be overlay these and finally display them. Its diverse applications include: (i) Campus Navigation - Layar tool is utilized to navigate campus with geo-location satellites, (ii) IGA 2017 Berlin - Layar was used to create a digital walk throughout the built area, albeit the system came with barriers. Therefore, RADAR-system (Resource Annotation and Delivery for Mobile Augmented Reality Services) was introduced to breach the barriers and permit the users to place their own location data. Even these apps were limited by the large streaming files and the availability/accessibility of the internet, (iii) Building Culture Saarbrunkrn - to overcome the limitations of the GPS based AR systems, alternative systems based on image recognition tracking systems are also used. "Layar" published a version known as "Layar vision", with its first application being tested in Saarbrunkn. The system was updated with the city street photos of 1950s, with colour marking the architectural elements that made the city legible and were in the pre-set time covered with hoardings or had been destroyed. The experiment was done at various points throughout the city to involve the local people and letting them experience the lost heritage and what the city aimed to get back, and (iv) Augmented Plan - when it involves the mediation of the plans that we present to the client, most of the information we lay in front is tough to comprehend. As a solution to this, apps can take the virtual data such as plan and augment it within the client's phone itself.

(Redondo et al. 2011) ${ }^{[22]}$ VR and AR research have shown great potential in the field of design education. However, not much progress has been made in the field of education through these tools, due to technological issues and device costs. In Architectural and urban design the understanding of spaces is an essential component. Currently, this knowledge is achieved during the latter stages of a professional's carrier, while with the help of AR the students could be introduced to these scenarios during the initial part of the course itself. It will result in better understanding for the students compared to the traditional tools used to study architecture that are still majorly limited to 2D. It is essential to explain the work process in architecture to juggle between multiple other disciplines to make a project feasible. Traditional representations embody both a need for abstraction and interpretation and can thereby be challenging to understand. Therefore, they possibly mislead those not trained in the field. In response to this, architectural projects and urban design drawings are complemented with perspectives, text and 3D models, both physical and digital. Still, there is a common problem of understanding both spatial qualities and the scale of proposed buildings and structures. As a result, project-owners, politicians, decision-makers, and the public often have expectations based on their interpretation that differ from or exceed the realised project's qualities in its context. In urban design, it becomes vital to have a human-spatial experience. Changes we need to look forward to (i) prior to realisation, complete 3D models of proposed projects can be studied regarding all aspects. Today we build 3d models, after AR we will be able to experience them and in future maybe make in situ changes, (ii) one can experience simulation of physical conditions changes, animate and visualize pollution, noise, etc., (iii) human factor studies will be made tangible and better outcomes for the design will be achieved, and (iv) public opinion and suggestions could be incorporated as through these tools, design can be accessible to all.

(Van Dessel, 2018-2019) ${ }^{[23]}$ Virtual Reality deals with a holistically created or designed models; it is well utilized with the augmented reality which uses real time location. Traditionally in urban design cardboard is used as a means to create physical models, which is not a very flexible option and the visualisation of the space is done only from a bird-eye view. City Model - creating a virtual model to immerse in the design and make changes along the visualisation is one of the 
main aims of the VR. The main advantage is to be able to see the proportions and height which otherwise cannot be visualised in the 2D GIS system, currently in use.

Three visualisation techniques have been adopted (1) - Photorealistic Visualisation aims at providing a real like impression of the environment, mainly for the non-experts. The complex geometrical forms of the environment e.g., trees increase the file size and create multiple issues. (2) - Visualisation of Information and Data which enables information to be comprehended and immersed with the city model e.g., occupancy, year of construction, etc. (3) - Illustrative and non-photorealistic visualisation can be used for creating the first look of the model, providing with the urban information, helping in decision making process.

City GML - it is a standard core model that acts as a source of information for multiple disciplines which require urban data. Although the standardised model does provide a clear structure, the coding languages are unknown to the urban planners. This becomes a little cumbersome and restrictive to the designers.

(Ahmed et al. 2019) ${ }^{[24]}$ The recent development in information technology has huge opportunities to improve the architectural education in terms of methodologies, strategies and tools. Studies explores the importance of using virtual reality with open minded and progressive methods in teaching architectural courses and not just stick to the traditional methods of yesteryears. The research aimed at evaluating the (BCIVR Software) in architectural education of building construction courses as a case study at Jordan University of Science and Technology (JUST). This Building Construction using Virtual Reality technology (BCIVR) is computer software designed by the authors for research purpose and presents 4D models (3D model and time dimension) for certain building construction phases using VR technology to do immersive and non-immersive virtual reality experience for the users in terms of three axes: (i) providing students with the building construction information, (ii) achieving enjoyment, and (iii) integrating with other courses. The data was obtained from architecture students on a structured questionnaire. The results were highly in favour of VR software than those of the traditional teaching methods. As conclusion, the use of BCIVR software as tool in building construction courses is very useful and effective for the students. The VR technology can also find application in many other courses offered in the curriculum of Architectural programs. VR integrates the traditional way of education by bringing the real world into the classroom, however, its application in architectural educational purposes have been limited so far.

(Gębczyńska-Janowicz, 2020) ${ }^{[25]}$ The evaluation of research presented in this article indicated its effectiveness as an educational tool in the architectural design studio to improve learning. Realistic concept visualization enables students to experience the special consequences of proposed solutions. VR is able to support the design of commemorative places by applying reconstructed buildings to the real image. With virtual reality, the guide could use tools to create an augmented reality experience, being able to draw attention to certain elements that otherwise may not be easy to explain in real life. CAVE is one such interesting tool for an immersive design environment. Virtual Reality (VR) technology allows architectural design classes based on advanced digital technologies, enabling a broad understanding of concept visualization. VR allows you to see experience from different points of view and can also be used to generate 3D images of architectural related elements, such as a parapet detail, to assist in quick and deeper learning experience in understanding architecture and construction. Previously, the key argument inhibiting the presence of year in education of architectural design was the cost of purchasing technology related equipment and the difficulty of acquiring skills to operate it. Today, students are fluent in the application of CAD software and graphics processing applications. Students who have experience in computer modeling are becoming increasingly familiar with VR which helps promote changes in both education and architectural practice. 
(Sardo et.al, 2018) ${ }^{[26]}$ Augmented reality is discussed as a tool that immerses a person into an altered reality by not only connecting with the sight and sound, but also explores in incorporating the other three senses which have not been as explored as the other two. The multisensory AR is believed to involve a user to experience the better involved experience. The paper develops the interface by centering on a museum, but some of these can be added on to enhance AR experience. The interface system experiments also concentrate on moving from the big computer setups to more portable computer systems that can connect with the phones. Simulation for these senses is experimented with users to get results such as the simulation of touch and feel are explored with vibration, thermal simulation and air flow. There are not many options to stimulate the taste sense, but it has been studied that with the help of sight and smell, the sense for taste can also be developed. Vaporization was also a technique utilized to simulate smell. These experiments utilized some previously existing techniques and enhanced them to achieve the desired results. The conceptual model had a great response from the user survey even though it had some shortcomings and can still have better outputs with improvements.

(Megahed, 2014) ${ }^{[27]}$ The use of AR is studied for the creative and perceptive development of the students in the field of design. The incorporation of these tools can shift the current teacheroriented learning to a student-oriented learning. However, this incorporation still faces a lot of technological and social challenges. The pedagogy for the real life lessons has been in 2-D for a long time while the real objects itself exist in 3-D. Architectural design education is a field which requires a lot of practical knowledge and new methods to evolve the creativity of the students, with the constant changes coming up in the field of design it becomes essential to adapt, incorporating new techniques. The initial stage of data collection and analysis while experiencing the environment of different cities while sitting in the classroom gives a clearer perspective of context. The early stages of a design process involves most of the brainstorming, this stage requires the freedom for visualization and to be able to communicate it at its best. The next stage which incorporates a lot of reviewing and redo's can be made easy with the tools as mistakes can be corrected in real time on the spot. The paper discusses the working of two software in the field of education i.e., ARTHUR and AR Workbench.

(Abramovici, 2017) ${ }^{[28]}$ Throughout the life-cycle of a building, maintaining the original conditions costs the most. The paper not only deals with visualizing the maintenance data but it also incorporates team wide communication and indicates any warning situations that require coordination. To make things efficient a co-ordination is developed amongst the machines and humans by using smart devices and internet of things. The whole concept is based on the graph model (GUI- Graph user interface) that analyses the type of goals and their approach to it. The model discussed is a cloud based system which requires the machines or objects requiring maintenance to be a part of the internet of things (cloud based system). The model connects with the individual technician leading to the activity that is related to other activities, the sensors record the results and values for follow up activities. The implementation of a prototype of the model was conducted to note the workability of the model, although the model could use more organized and a holistic planning. The author is now onto the stage of proof of concept.

\section{DISCUSSION}

$\mathrm{AR}$ and VR as tools for architecture and urban design have been theorized conceptualized since 1967 , with the first research paper published by Schutz and North-Western University $(1967)^{[2]}$. Since then, various advancements have been made and AR started to be used in indoor settings while VR was used for visualization. From then to now, the progress in the workability and application of these tools has been very slow paced, due to the reason that this technology has not been developed exclusively to suit this particular field. However in recent years, certain software's and hardware's have been developed for the specific use in the design field, which 
explored immersive communication, real time visualization and data analysis. The scope of these tools has been explored more in concepts and experiments that work with the potential of the technology being used to (i) create an immersive user interaction, which creates a presence of your design and immerses you into the project to experience the impact of the project. (ii) To improve the real time positioning and tracking system. (iii) Provides the user to experience the design well before construction which enables to incorporate feedback. (iv) Incorporating these tools as part of an education system will expose the students to practical issues well before becoming proper architects. (v) Communicating designs to the workers is an issue that leads to a lot of causalities and management problems that can be life threatening, but can be inverted by using AR and VR to foresee these issues. (vi) Deteriorated/dilapidated heritage buildings with time could be experienced and visualized. (vii) Also, the complex urban fabric of the modern era, where all buildings are growing sky high and loosing individuality, becoming tedious to navigate around the city, for them these visual aided tool could work wonders.

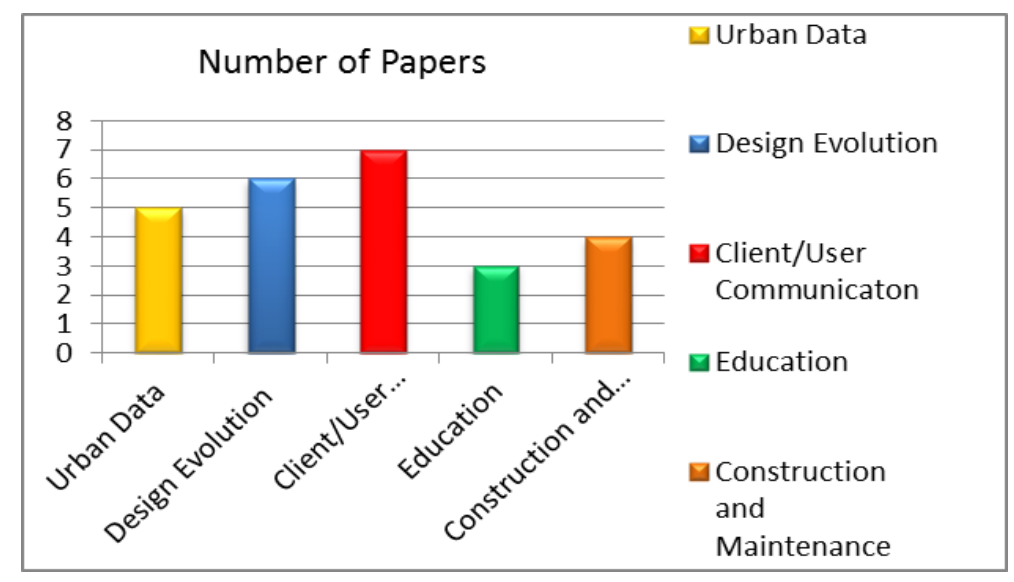

Figure 1. Categorization of AR/VR under diverse sub heads of architecture \& urban design

However, these tools still come with a number of limitations in the field of technology and economics. The tools need to be more users friendly, accessible and economically viable to all the professionals and end-users. The industrial production of these tools can be seen taking place only after overcoming the limitations.

\section{Conclusion}

The article synthesizes the wide variety of architecture and urban design options that are being developed, conceptualized or is currently in use in the field of Augmented Reality (AR) and Virtual Reality (VR). The research papers briefly describe the diverse avenues that have the scope of development or improvement under AR. Collaboration and immersion are two aspects that are being explored in the field of design. With the complexities in design introduced by evolving time it becomes essential to manage this data in an efficient manner to seek maximum output. Reading, studying and sketching are important for understanding and designing for the people, but with AR and VR a fourth dimension is added to our comprehension. Human Computer interface is at the beginning of its era and it has the potential to change the depth of our understanding about the spaces and its ergonomics. Presently, the architects are already familiar with CAD tools and various simulation, analysis and management tools which typical VR technologies are allowing architectural design to be virtually represented and realised before construction. AR takes things to the next level and allows these virtual visualisations to be inserted into the physical reality. Thus, the ongoing future developments of AR and VR will play a significant role on improving the design of the built environment. 


\section{ACKNOWLEDGEMENT}

I would like to place my thanks to Prof (Dr) Madhura Yadav, Director, School of Architecture and Design, Manipal University, Jaipur for her guidance. I also want to thank Dr. Vaneet Jishtu, Scientist, HFRI for help in motivation and guidance.

\section{REFERENCES}

[1] Husserl, E and Gibson, WRB 1931 Ideas: general introduction to pure phenomenology, G. Allen \& Unwin Ltd., London. Pp. 465.

[2] Schutz, A and Northwestern University (Evanston Ill.) 1967 The phenomenology of the social world, Northwestern University Press, Evanston, III.

[3] Norman, D. 1988. The Psychology of Everyday Things, Basic Books, New York.

[4] Frazer, JH, Frazer, JM and Frazer, PA 1980 Intelligent Physical Three-Dimensional Modelling Systems, Computer Graphics 80 Conference, pp. 359-370.

[5] Seichter, Hartmut and Schnabel, Marc Aurel 2005 Digital and Tangible Sensation:

[6] Sørensen, Søren S 2006The development ofAugmented Reality as a tool inArchitectural and Urban designNordic Journal of Architectural Research. Volume 19, No 4, 2006.

[7] Döllner Jürgen, Konstantin Baumann, Henrik Buchholz 2006 Techniques and strategies of visualization complex 3D spatial data. CORP 2006 \& Geomultimedia06. Assessed on 12.011.2020.

[8] Seichter, H 2007 Augmented reality aided design. (Thesis). University of Hong Kong, Pokfulam, Hong Kong SAR. Retrieved from http://dx.doi.org/10.5353/th_b3896659

[9] Wang, Xiangyu (2009). Augmented Reality in Architecture and Design: Potentials and Challenges for Application. International Journal of Architectural Computing. 2009;7(2):309-326. doi:10.1260/147807709788921985.

[10] Abboud, Rana (2014). Architecture in an Age of Augmented Reality:Opportunities and Obstacles for Mobile AR in Design, Construction, and Post-Completion.

[11] Seichter, Hartmut 2020 Benchworks - Augmented Reality Urban Design. CAADRIA (2004). [Proceedings of the 9th International Conference on Computer Aided Architectural Design Research in Asia / ISBN 89-7141-648-3] Seoul Korea 28-30 April 2004, pp. 937-946.

[12] ARtoolkit. http://www.hitl.washington.edu/home/. Assessed on 15.11.2020.

[13] Kim, Mi jeong \& Wang, Xiangyu \& Zhu, Xingquan \& Kang, Shih-Chung (2011). Augmented Reality Research for Architecture and Design. 10.4018/978-1-61350-180-1.ch013.

[14] Chung, DHJ, Zhiying, SZ, Karlekar, J, Schneider, M, \& Lu, W (2009). Outdoor Mobile Augmented Reality for Past and Future On-Site Architectural Visualizations, Computer Aided Architectural Design Futures. pp.557-571.

[15] Wang, X, \& Dunston, PS (2005). Real Time Polygonal Data Integration of Cad/Augmented Reality In Architectural Design Visualization, Computing in Civil Engineering (American Society of Civil Engineers, $A S C E$ ) (pp.1-8).

[16] Santos, P, Gierlinger, T, Stork, A \& McIntyre, D (2007). Display and Renering Technologies for Virtual and Mixed Reality Design Review.Interational Conference on Construction Applications of Virtual Reality. pp.165-175.

[17] Belcher, D, \& Johnson, B (2008). MxR: A Physical Model-Based Mixed Reality Interface for Design Collaboration, Simulation, Visualization and Form Generation.Association for Computer Aided Design in Architecture. pp. 464-471.

[18] SchubertGerhard, David Schattel, Marcus Tönnis, Gudrun Klinker, Frank Petzold (2015). Tangible mixed reality on-site Interactive augmented visualisations from architectural working models in urban design. São Paulo, Brazil : s.n., 2015. The Next City - New Technologies and the Future of the Built Environment $-16^{\text {th }}$ International Conference. pp 55-74.

[19] Thabet, Walid \& Shiratuddin, Mohd Fairuz \& Bowman, Doug (2002). Virtual Reality in Construction. In: Engineering Computational Technology. pp. 25-52. Publisher: Saxe-Coburg Publications. March 2002, updated 2015.

[20] Milovanovic Julie, Guillaume Moreau, Daniel Siret, Francis Miguet (2017). Virtual and Augmented Reality in Architectural Design and Education: An Immersive Multimodal Platform to Support Architectural Pedagogy. 17th International Conference, CAAD Futures, Gülen Çağdaş, Mine Özkar, Leman F. Gül and Ethem Gürer, Jul 2017, Istanbul, Turkey. 
[21] Broschart, Daniel \& Zeile, Peter (2014). Architecture - Augmented Reality Techniques and Use Cases in Architecture and Urban Planning. [https://www.researchgate.net/publication/269574525] Updated Feburary 2018.

[22] Redondo, Ernest \& Navarro, Isidro \& Riera, Albert \& Fonseca, David (2011). Visual Interfaces and User Experience: Augmented Reality for Architectural Education: One Study Case and Work in Progress. Communications in Computer and Information Science. 166. pp. 355-367.

[23] Anke, Van Dessel (2019). The Use of Virtual Reality in Urban Planning Review paper on the (dis)advantages and a new perspective for future research. Master thesis - Master of Urbanism and Spatial Planning. Center for Urban Development, University of Antwerp.

[24] Ahmad K. Bashabsheh, Hussain H. Alzoubi, Mostafa Z. Ali (2019). The application of virtual reality technology in architectural pedagogy for building constructions. Alexandria Engineering Journal,Volume 58, Issue 2. pp. 713-723. ISSN 1110-0168. https://doi.org/10.1016/j.aej.2019.06.002.

[25] Gębczyńska-Janowicz, Agnieszka (2020). Virtual reality technology in architectural education . World Transactions on Engineering and Technology Education. Vol. 18. No. 1. http://www.wiete.com.au/journals/WTE\&TE/Pages/Vol.18,\%20No.1\%20(2020)/04-GebczynskaJanowicz-A.pdf

[26] Sardo, J.D., Pereira, J.A., Veiga, R., Semião, J., Cardoso, P., \& Rodrigues, J. (2018). A Portable Device for Five Sense Augmented Reality Experiences in Museums.

[27] Megahed. N. A. (2014). Augmented Reality Based - Learning Assistant for Architectural education.

[28] Michael Abramovici, Mario Wolf, Stefan Adwernat, Matthias Neges (2017). Context-aware Maintenance Support for Augmented Reality Assistance and Synchronous Multi-user Collaboration, Procedia CIRP, Volume 59; pp. 18-22

\section{AUTHORS}

Pearl Jishtu Presently pursuing her final year in Bachelor of Architecture, at Manipal University Jaipur. Finds motivation towards research work, in the role of technology in architecture. She has held positions of responsibility and has represented the University at National Association of Students of Architecture.

Prof (Dr) Madhura A Yadav An Architect-Planner with over 25-year experience. She is Founder Head of the School Architecture \& Design and now Director of SA\&D at Manipal University Jaipur. She is one of the founder members in establishing Faculty of Design at Manipal University Jaipur and founder head for fashion Design, Interior Design and Planning programmes. She is/was on several Committee/Expert Groups set up by Government of India., She is on Jury of various Architectural competitions. She is also on Editorial Board Architecture, Urban Design and Urban

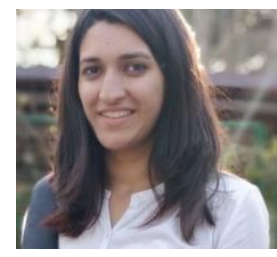
Planning India Journals. She has published number of papers in national and international journals and conferences. She is UNESCO certified Mentor for Community based participatory research. She is Indian Green Building Accredited Professional. She has conducted workshops/conferences on Waste Management, Bamboo Architecture, Earthquake Resistant Structures, Sustainable Cities/ buildings etc. Her current research interests are Architecture- Ecology interface and Eco- cities, Promotion of eco materials and Community Based Participatory Research. She is recipient of Distinguished Faculty in Architecture Award 2017 and Educational Leadership Award 2019.

(C) 2020 By AIRCC Publishing Corporation. This article is published under the Creative Commons Attribution (CC BY) license. 